\title{
Bis(benzylamine) monomers: One-pot preparation and application in dendrimer scaffolds for removing pyrene from aqueous environments
}

\author{
Olivia N. Monaco, Sarah C. Tomas, Meghan K. Kirrane and Amy M. Balija*
}

\section{Full Research Paper}

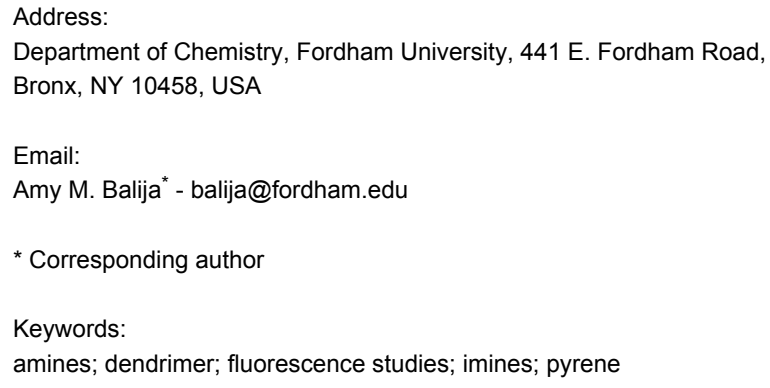

Beilstein J. Org. Chem. 2013, 9, 2320-2327.

doi:10.3762/bjoc.9.266

Received: 01 July 2013

Accepted: 07 October 2013

Published: 31 October 2013

Associate Editor: H. Ritter

(c) 2013 Monaco et al; licensee Beilstein-Institut. License and terms: see end of document.

\begin{abstract}
Bisimine and bisamine $\mathrm{AB}_{2}$ monomers have been synthesized from 3,5-diaminobenzoic acid and benzaldehyde derivatives without the need for protective groups or purification. This monomer preparation is universal for various electron-donating and electronwithdrawing benzaldehyde substrates. To demonstrate the versatility of these previously unreported $\mathrm{AB}_{2}$ monomers in the formation of high molecular weight structures, novel first-generation dendrimers and hybrid second-generation dendrimers have been synthesized. Using fluorescence spectroscopy, pyrene was shown to be removed from an aqueous environment upon exposure to thin dendrimer films, with the first-generation dendrimer removing $70 \%$ of the pyrene within $30 \mathrm{~min}$ and the hybrid second-generation dendrimers removing $38-52 \%$. Inclusion formation constants were calculated to be on the order of $10^{9}-10^{11} \mathrm{M}^{-1}$ and are comparable to the values of previously reported macromolecules. These results illustrate that size may not influence pyrene removal as effectively as composition.
\end{abstract}

\section{Introduction}

Highly-branched polymeric systems provide an attractive route for removing pollutants from water due to their interior cavities and their ease of formation [1]. While several promising approaches have been reported [2], these polymeric waterpurification systems utilize a patching method, in which the periphery of known architectures is modified with specific functionalities for water treatment processes. Alternatively, dendrimers $[3,4]$ provide the branched architecture while main- taining the ability to incorporate functional groups at precise locations, which may be ideal for removing specific pollutants. However, the use of dendrimers may be hindered without the development of new, more convenient approaches to prepare these systems with minimal purification [5]. Although several strategies offer solutions towards preparing dendrimers more efficiently [6-10], these methods are limited to specific functionalities. There remains an unmet need for novel, flexible syn- 
thetic pathways to broaden the functional groups utilized to fine-tune the periphery, core, and branching sites of dendrimers.

The objective of the research disclosed is the development of a straightforward synthesis for novel dendrimers that can effectively remove small organic molecule pollutants from water. Furthermore, the ability to fine-tune the dendrimer easily is desired. The focus of this report is on three areas of progress: (1) the synthesis of new $\mathrm{AB}_{2}$ monomers without the use of protective groups and with minimal purification; (2) the incorporation of these monomers into dendrimeric architectures; and (3) the examination of how effectively these new dendrimers remove a representative organic pollutant from an aqueous solution.

\section{Results and Discussion}

Condensation of 3,5-diamino benzoic acid (1) with 2.5 equiv of benzaldehyde (2a) in methanol at room temperature resulted in the precipitation of bisimine monomer $\mathbf{3 a}$, which was isolated cleanly by filtration after 20 minutes $[11,12]$. Electron-deficient, electron-rich, and sterically hindered benzaldehydes $\mathbf{2} \mathbf{b}-\mathbf{g}$ could also be utilized to prepare the corresponding bisimine products (Table 1, compounds 3b-g). Minimal impact on the overall yield of the reaction was observed upon varying the benzaldehyde concentration or substituting absolute ethanol for methanol; however, elevated temperatures hindered the ability to cleanly obtain bisimines 3 .
Alternatively, the condensation of 3,5-diamino benzoic acid with 2.1 equiv of benzaldehyde (2a) in methanol at room temperature followed by in-situ reduction with $\mathrm{NaBH}_{4}$ and acidification with $2 \mathrm{~N} \mathrm{HCl}$ resulted in the precipitation of bisamine 4a. Bisamine products were obtained with electrondeficient, electron-rich, and sterically hindered benzaldehydes (Table 2, Compounds $\mathbf{4 b}-\mathbf{h}$ ). The formation of $\mathbf{4 f}$ was difficult, resulting in lower overall yields and longer reaction times, possibly due to the electron-withdrawing properties of the nitro group [13]. Replacing $\mathrm{NaBH}_{4}$ with other hydride sources such as $\mathrm{NaBH}_{3} \mathrm{CN}$ did not impact the reduction rate of $\mathbf{4 f}$. To ensure a clean isolation of bisamines $\mathbf{4}$, the benzaldehyde starting material needed to be free from oxidized aldehyde byproducts. Using elevated temperatures to promote the formation of bisamines 4 was unsuccessful. This result was in contrast to comparable systems [14], which required heat to form benzyl amine products exclusively. A preliminary examination using ${ }^{1} \mathrm{H}$ NMR spectroscopy suggested that twelve hours were needed to completely reduce the proposed intermediate imine 3 . A mixture of products was obtained when $\mathbf{1}$ was replaced with methyl 3,5-diaminobenzoate.

The single-pot process of preparing derivatized bisimine and bisamine products from 3,5-diaminobenzoic acid (1) and benzaldehyde derivatives eliminates the use of protective groups, removes the need for purification, and follows the general principles of atom economy [15]. This simple one-step

Table 1: Formation of bisimine compounds $\mathbf{3 a - g}$.

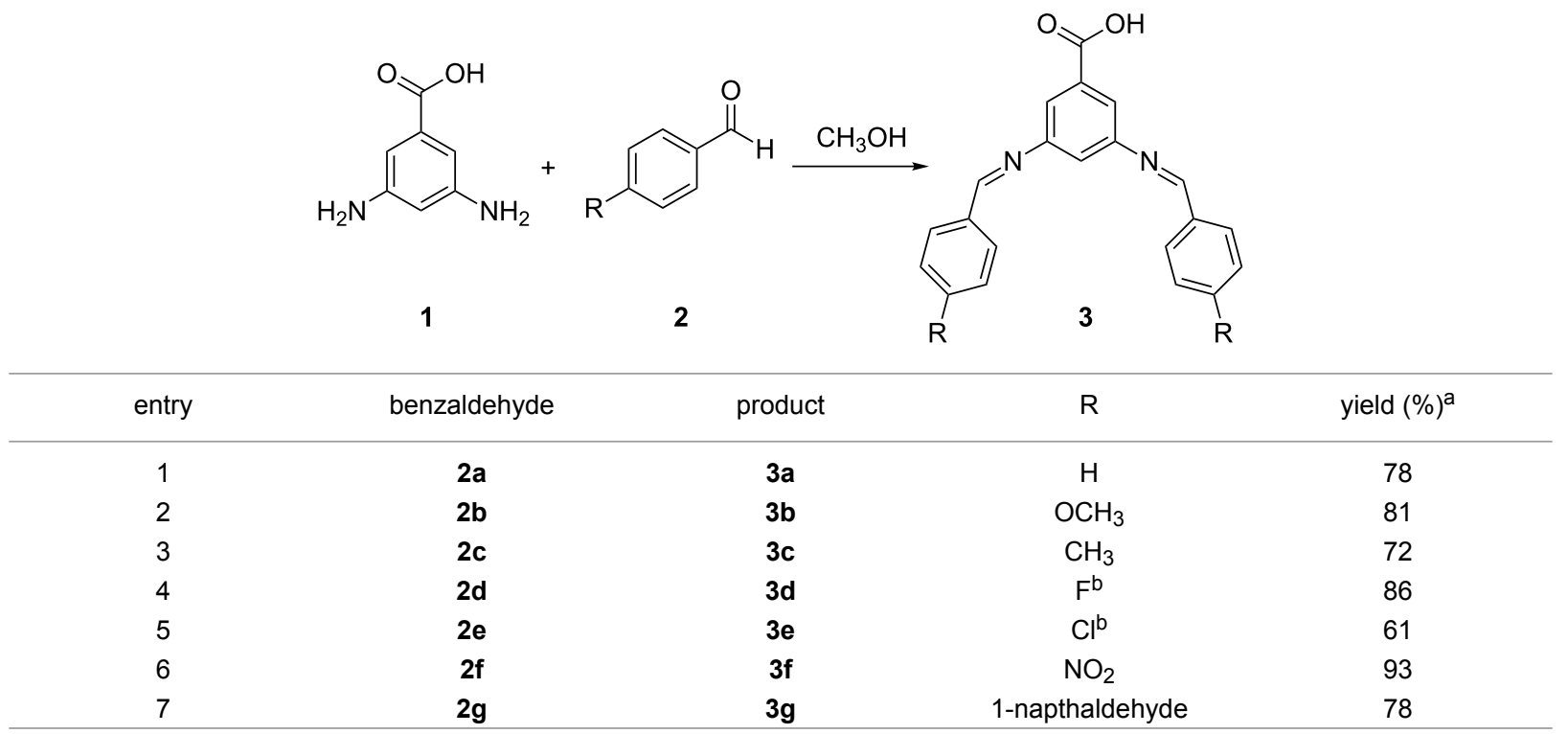

asolated yields. ${ }^{\text {b }} 90 \%$ pure by ${ }^{1} \mathrm{H}$ NMR spectroscopy. 
Table 2: Formation of bisamine compounds $4 a-h$.

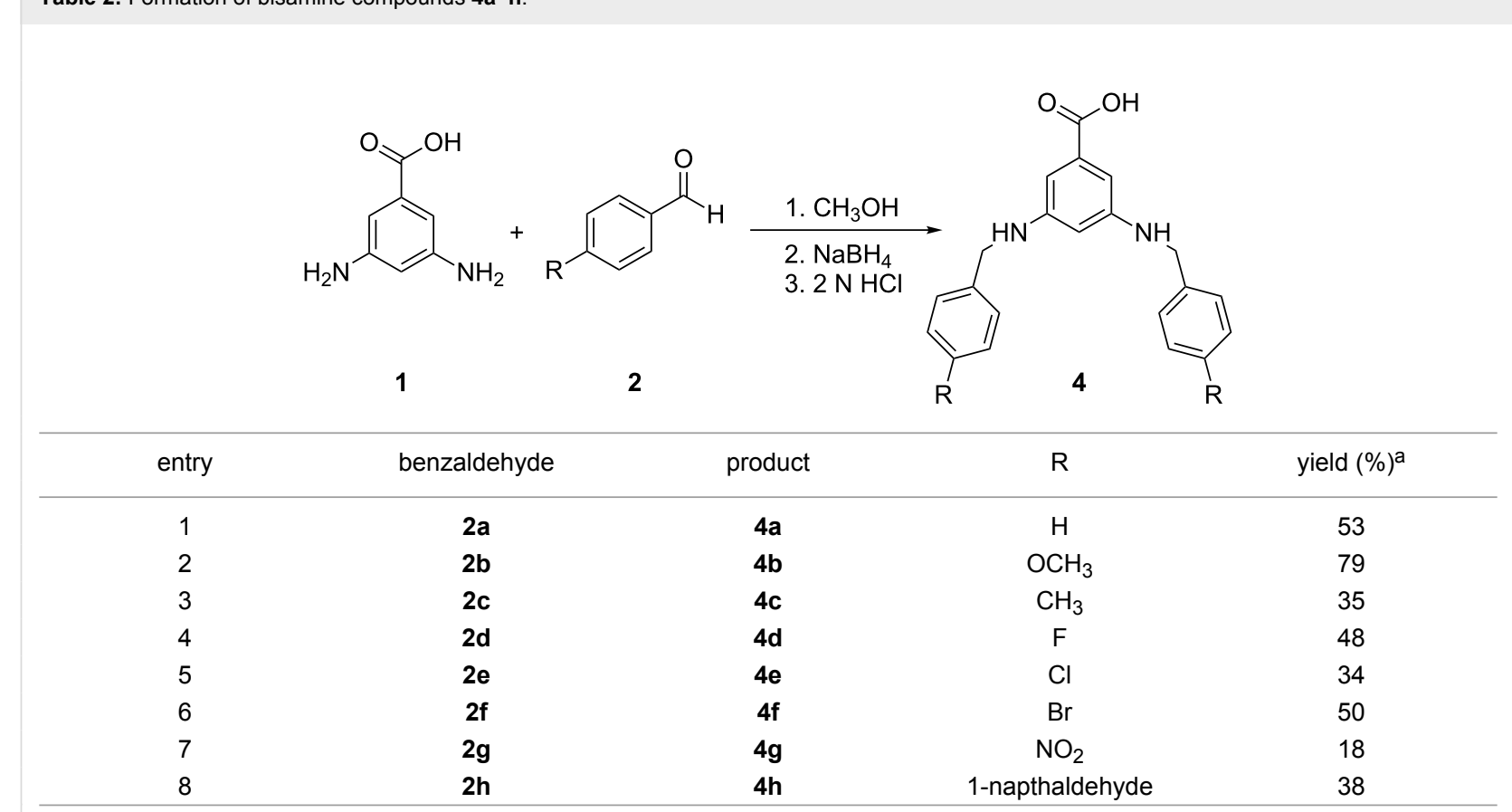

aproduct precipitated out of solution upon addition of $2 \mathrm{~N} \mathrm{HCl}$. The solid was filtered and washed with cold methanol.

approach is in contrast to the synthesis of the oxygen analogue of $4 \mathbf{a}$, the well-known $\mathrm{AB}_{2}$ bis(benzyl) ether monomer, which is prepared in three steps from 3,5-dihydroxybenzoic acid and benzyl bromide. When synthesizing the bis(benzyl) ether monomer, the carboxylic acid group must be masked, and the intermediates must be purified through column chromatography [16]. Furthermore, although many benzyl bromide compounds are commercially available, they are typically lachrymators and are considered hazardous as compared with their benzaldehyde counterparts. Therefore, the bisimine and bisamine synthesis disclosed in this paper are ideal for the generation of functionalized systems with a higher molecular weight without the use of hazardous reagents or generating large quantities of waste as compared with previously published systems.

Bisimine 3a was stable to ambient conditions in air over four months but degraded to release benzaldehyde within two hours in $\mathrm{CHCl}_{3}, \mathrm{CH}_{2} \mathrm{Cl}_{2}$, and THF as noted by the red shift in the UV-vis absorption spectrum. ${ }^{1} \mathrm{H}$ NMR spectroscopic analysis confirmed the disappearance of the imine peak upon exposure of bisimine 3 to acid. Hydrolysis occurred at different rates with varying functionalities on $\mathbf{3}$; however, a quantitative comparison of how the substituents affected hydrolysis could not be adequately obtained. Bisamine $\mathbf{4}$ was found to be more stable in air although it began to decompose after exposure to organic solvents for one hour.
While various applications are possible with monomers $\mathbf{3}$ and $\mathbf{4}$, the $\mathrm{AB}_{2}$ structure of these compounds is ideal for incorporation into dendritic systems $[17,18]$. As a proof of concept, the preparation of first-generation dendrimers was attempted with monomers 3a and 4a. Although imine-based dendrimers had been previously reported [19,20], bisimine 3a readily degraded to its starting materials under the condensation conditions necessary to form the dendrimer. Alternatively, the bisamine scaffold 4 was proposed to be superior to monomer 3 for incorporation into dendrimers due to its improved stability. Condensation of $\mathbf{4 a}$ with triphenol core 5 [16] resulted in the formation of first-generation dendrimer 6 (Scheme 1). Though successfully prepared, the dendrimer decomposed in most organic solvents, making it difficult to purify and analyze. Attempts to prepare other first-generation dendrimers were not pursued due the perceived instability.

To obtain a more stable system, 3,5-bis(benzyloxy)benzaldehyde 7 was designed such that upon condensation with 3,5diaminobenzoic acid, a hybrid bisamine/benzyl ether product would be obtained. It was proposed that the benzyl ether groups would mask the bisamine functionalities and result in increased compound stability. Structure 7 was successfully prepared through a multi-step synthetic scheme [21]. Condensation of $\mathbf{1}$ with 2.1 equivalents of benzaldehyde 7 followed by in situ reduction with $\mathrm{NaBH}_{4}$ and acidification with $2 \mathrm{~N} \mathrm{HCl}$ provided 


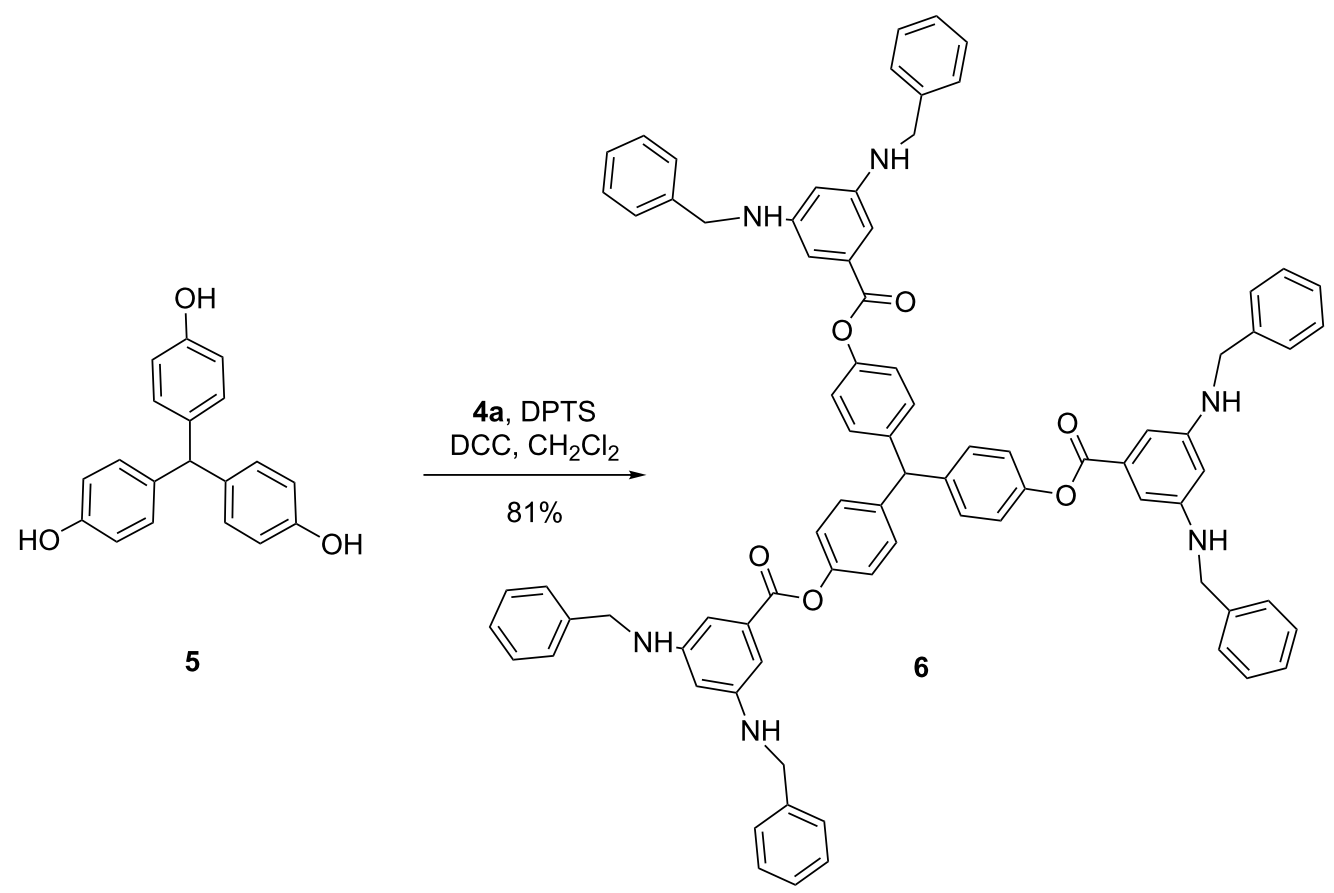

Scheme 1: Synthesis of first generation bisamine based dendrimer 6 .

dendron 8 (Scheme 2). Compound $\mathbf{8}$, itself an $\mathrm{AB}_{2}$ monomer similar to compounds $\mathbf{4 a}-\mathbf{4 g}$, was stable in $\mathrm{CDCl}_{3}$ as visualized by ${ }^{1} \mathrm{H}$ NMR spectroscopy.

Due to the improved stability of $\mathbf{8}$ in organic solvents, secondgeneration dendrimers containing the dendron and triphenol cores 5, 9, and $\mathbf{1 0}$ (Schemes 1 and 3) were designed. The triphenol cores were utilized to examine how the core diameter and shape influenced the chemical and physical properties of the resulting dendrimers. Cores $\mathbf{5}$ and $\mathbf{9}$ were calculated to exist in a helical conformation with an average diameter of $5.7 \AA$ while $\mathbf{1 0}$ was present as a planar structure with an average diameter of $2.1 \AA$ [22]. It was proposed that the cores with higher molecular weight would influence the overall properties of the corresponding dendrimer more than the smaller core. Standard coupling conditions [16] of 8 with triphenol cores 5, 9 and 10 resulted in second-generation dendrimers 11-13 (Scheme 3 ), which were successfully purified by size exclusion chromatography (SEC). The products were determined to be $>90 \%$ pure through ${ }^{1} \mathrm{H}$ NMR spectroscopy and their molecular weights were confirmed by MALDI-TOF. The resulting novel dendrimers contained six amino groups within the interior and twelve ether functionalities along the periphery.

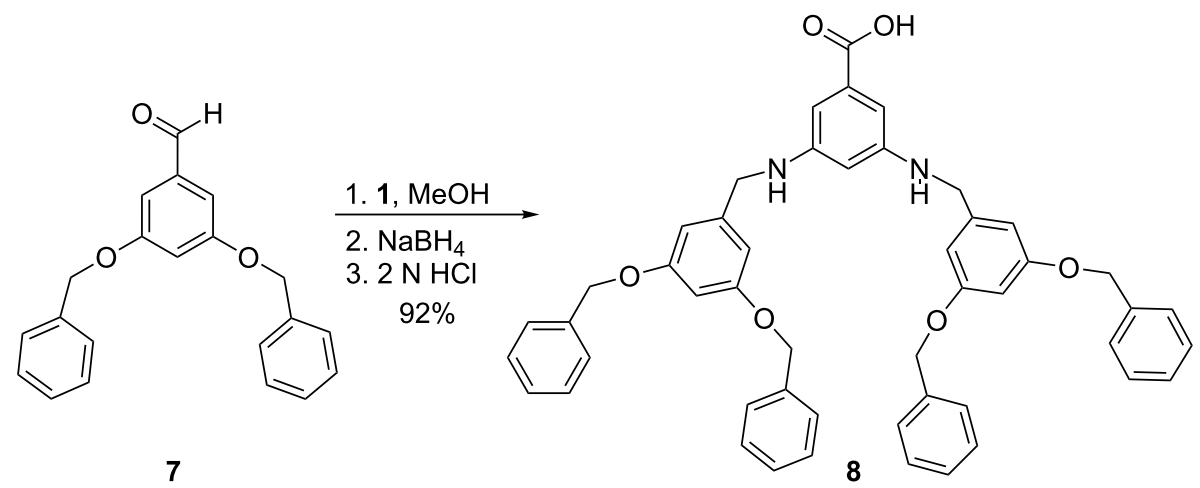

Scheme 2: Synthesis of dendron 8. 

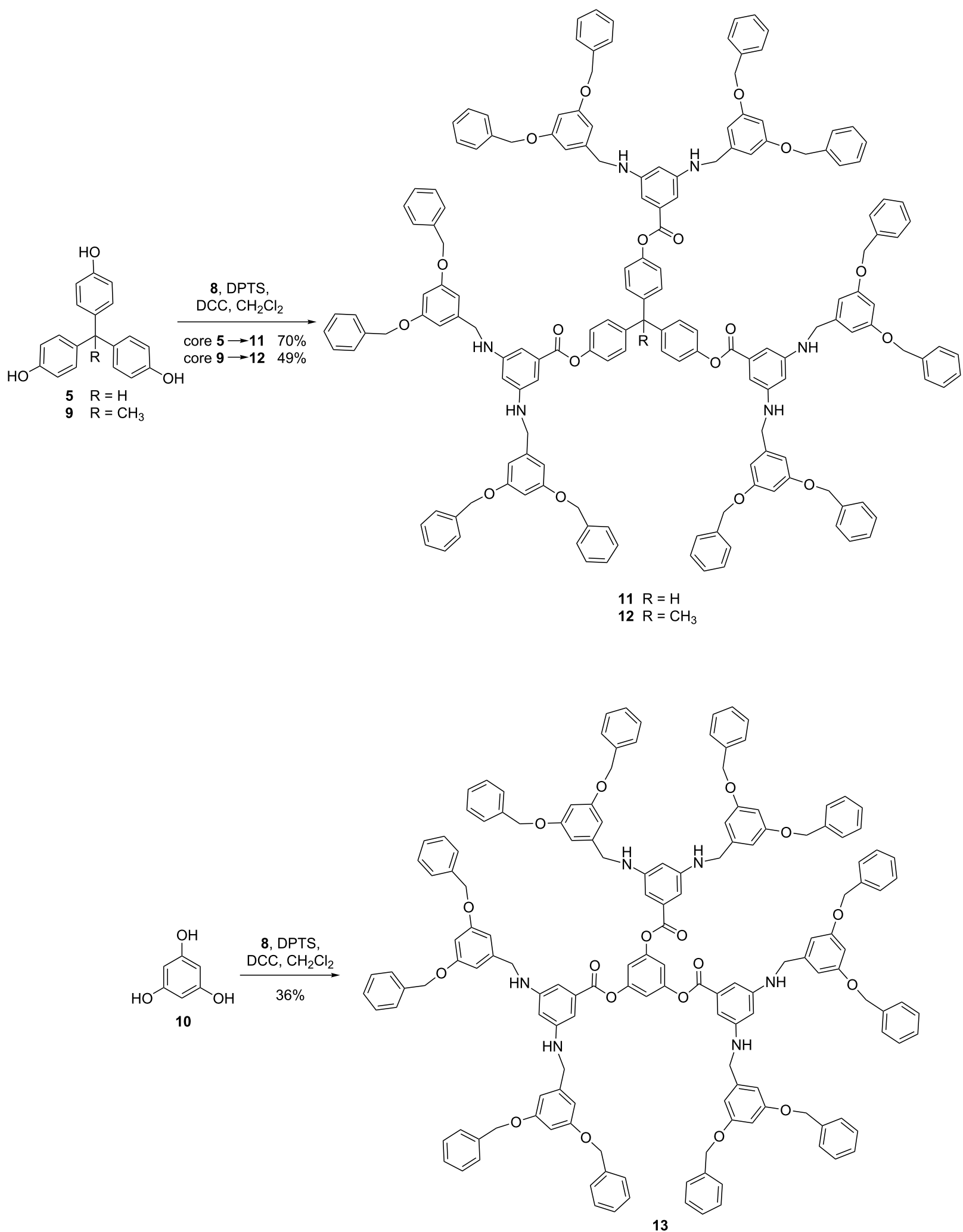

Scheme 3: Synthesis of hybrid dendrimers 11-13. 
Studies then were performed to determine whether the dendrimers could remain intact upon exposure to various external conditions. To investigate their stability in organic solvents, dendrimers 11-13 were separately dissolved in chloroform, and their ${ }^{1} \mathrm{H}$ NMR spectra were obtained. While the firstgeneration dendrimer $\mathbf{6}$ decomposed within one hour, the second-generation dendrimers 11-13 were stable under similar conditions. Additional studies focused on the stability of the dendrimers in water at $\mathrm{pH}$ 7. Thin solid films of $\mathbf{6}$ and $\mathbf{1 1}$ were prepared on the bottom of a beaker and water at $\mathrm{pH} 7$ was added to the beaker. After three days, the water was removed and the ${ }^{1} \mathrm{H}$ NMR spectra were obtained. No changes in the spectra were observed, which indicated that the dendrimers remained intact in an aqueous solution. Therefore, pure secondgeneration hybrid dendrimers could be isolated and used in different solvent environments without being degraded.

Due to their hydrophobic periphery and larger size, hybrid dendrimers 11-13 were envisioned as potential scaffolds to encapsulate small molecule organic pollutants such as polycyclic aromatic hydrocarbons (PAHs) [23-25]. The presence of PAHs in drinking water is a growing concern due to their carcinogenic properties and persistence in the environment [26,27]. Current methods to remove these hydrocarbons are becoming ineffective. To examine the ability of the hybrid dendrimers to remove small organic pollutants, saturated aqueous solutions of pyrene [28], a representative $\mathrm{PAH}$, were introduced to beakers containing thin films of the hydrophobic dendrimers $[29,30]$. Aliquots were taken at $30 \mathrm{~min}, 60 \mathrm{~min}$, and two days, and the pyrene fluorescence intensity was determined by fluorescence spectroscopy [31]. After $30 \mathrm{~min}$, a 38-52\% decrease in the pyrene fluorescence intensity was recorded with dendrimers 11-13 (Figure 1), a change that was not observed in the absence of the dendrimers.

The intensity of the pyrene fluorescence continued to decrease upon longer exposure to the dendrimer films. After two days, dendrimers 11 and 12, which contained the cores with larger diameters, showed a $95 \%$ decrease in pyrene fluorescence intensity, while the exposure to dendrimer 13 resulted in a 70\% reduction. No additional decrease in the fluorescence intensity of pyrene was observed over time. As a comparison, the expo-

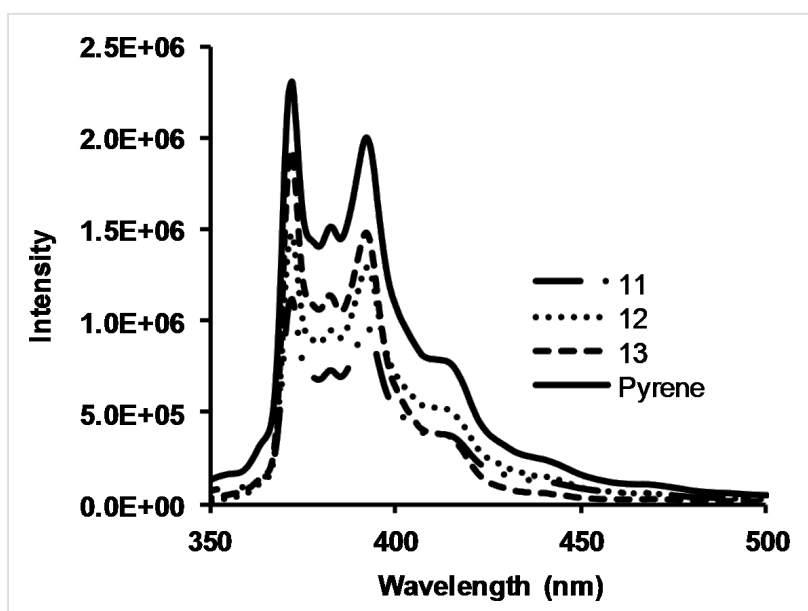

Figure 1: Fluorescence of a saturated aqueous solution of pyrene after $30 \mathrm{~min}$ exposure to dendrimers 11-13.

sure of first-generation dendrimer 6 to a saturated aqueous pyrene solution lead to a $74 \%$ decrease in pyrene fluorescence intensity after 30 minutes and $90 \%$ decrease in pyrene fluorescence intensity after two days.

This decrease in the pyrene fluorescence intensity upon exposure to the dendrimers may suggest that a hydrophobic, nonspecific interaction is occurring between the pyrene and dendrimer film. Over time, the dendrimer film becomes saturated with the pyrene, resulting in its inability to remove additional molecules. Although the size of the dendrimer core did not dramatically influence the pyrene fluorescence signal after $30 \mathrm{~min}$ in dendrimer $\mathbf{1 3}$, the smaller-core dendrimer was not as effective over two days. Furthermore, first-generation dendrimer 6 resulted in a higher initial change in the pyrene signal, which is hypothesized to result from the greater density of amine groups, which could favorably interact with the PAHs [32].

Pyrene inclusion formation constants were calculated using the measurement results obtained with the thin film experiments after two days (Table 3). The inclusion values were large, ranging from $10^{9}$ to $10^{11} \mathrm{M}^{-1}$, and are comparable to previous results obtained with cyclodextrin polymers $\left(10^{9} \mathrm{M}^{-1}\right)$ [33] and alkylated fifth-generation diaminobutane poly(propylene imine)

Table 3: Inclusion formation constants $K\left(\mathrm{M}^{-1}\right)$ and Gibbs free energies $\Delta G^{\circ}(\mathrm{kcal} / \mathrm{mol})$ of dendrimers 6 and 11-13 and pyrene.

\begin{tabular}{lllll} 
entry & compound & $K\left(\mathrm{M}^{-1}\right)$ & $\Delta G^{\circ}(\mathrm{kcal} / \mathrm{mol})$ & capacity (mol pyrene/g dendrimer) \\
\hline 1 & $\mathbf{6}$ & $2.44 \times 10^{10}$ & -14.1 & $6.34 \times 10^{-7}$ \\
2 & 11 & $1.02 \times 10^{11}$ & -15.0 & $3.12 \times 10^{-7}$ \\
3 & 12 & $1.62 \times 10^{11}$ & -15.3 & $3.31 \times 10^{-7}$ \\
4 & 13 & $6.09 \times 10^{9}$ & -13.3 & $3.34 \times 10^{-7}$
\end{tabular}


dendrimers $\left(10^{8} \mathrm{M}^{-1}\right)$ [28-30]. The large magnitude is proposed to be due to the thermodynamically favorable process of pyrene moving from a hydrophilic to a hydrophobic environment. A similar conclusion can be drawn from calculating the Gibbs free energies of each system, which lie between -13 and $-15 \mathrm{kcal} /$ mol and further demonstrate the favorable interaction of the dendrimers with pyrene in an aqueous environment $[29,30]$. The capacity of the dendrimers to remove pyrene was estimated to be between $3.12 \times 10^{-7}$ to $6.34 \times 10^{-7} \mathrm{~mol}$ of pyrene per gram of dendrimer. Current levels of pyrene in industrialized countries range from $1.48 \times 10^{-12}$ to $1.98 \times 10^{-10} \mathrm{~mol}$ of pyrene per liter of water [34]. Qualitatively, relatively small amounts of dendrimer $(<1 \mathrm{~g})$ should effectively remove pyrene from $1 \mathrm{~L}$ of stagnant water.

Overall, the relatively small dendrimers $\mathbf{6}$ and 11-13 gave similar results compared to polymers with higher molecular weight and therefore, large polymeric systems may not be necessary to effectively remove PAHs from water. Furthermore, the flexible preparation of $\mathrm{AB}_{2}$ bisamine monomers using different benzaldehyde starting materials allows dendrimer properties to be readily tuned to remove pollutants more effectively. This is more difficult to accomplish with commercially available dendrimers and polymeric systems. Although not applicable for large scale production due to its instability in organic solvents, first-generation dendrimer $\mathbf{6}$ does illustrate the importance of the bisamine monomer in removing pyrene.

\section{Conclusion}

Described is the synthesis of bisimine and bisamine $\mathrm{AB}_{2}$ monomers through a one-step process, which requires no purification and no manipulations of protective groups. Unlike the analogous benzyl ether $\mathrm{AB}_{2}$ compounds previously reported, these monomers were formed at room temperature and were filtered and washed to obtain the desired product. The reaction was found to be general for aromatic aldehydes except strong electron-donating benzaldehydes that resulted in unstable imines. An unprecedented first-generation bisamine-based dendrimer $\mathbf{6}$ was prepared but was found to degrade readily in organic solvents. More stable second-generation dendrimers composed of hybrid dendrons 8 and one of the three tri-phenol cores $\mathbf{5}, \mathbf{9}$, and $\mathbf{1 0}$ were synthesized.

One application for these novel structures is the removal of polycyclic aromatic hydrocarbons, such as pyrene, from water. Upon exposure of an aqueous solution of pyrene to the thin dendrimer film, no significant difference in the pyrene fluorescence intensity was observed after $30 \mathrm{~min}$ for the hybrid dendrimers 11-13, regardless of the core composition. After two days, the second-generation dendrimers that contain the larger cores 5 and $\mathbf{9}$ were more efficient in removing pyrene relative to core 10. While the larger dendrimers were effective at removing pyrene over two days, the first-generation dendrimer 6 extracted more pyrene after $30 \mathrm{~min}$ than 11-13. Pyrene inclusion constants for the dendrimers 6 and 11-13 were comparable to other polymeric purification systems, suggesting that large polymeric systems are not necessary to remove pyrene. Rather, the composition of the purification system appears to play an important role. Current efforts are focused on incorporating the bisamine dendron motif into additional supramolecular structures such as star polymers and hyperbranched systems and examining how fine-tuning the steric and electronic nature of the bisamine $\mathrm{AB}_{2}$ monomers influences the removal of pyrene from water. The results of these studies will be reported in due course.

\section{Supporting Information}

\section{Supporting Information File 1}

Full experimental synthetic procedures for compounds

3a-g, 4a-h,6-8, and 11-13, and pyrene fluorescence spectral data.

[http://www.beilstein-journals.org/bjoc/content/ supplementary/1860-5397-9-266-S1.pdf]

\section{Acknowledgements}

We thank Tom Carberry and Marcus Weck from New York University for their help in obtaining the MALDI-TOF data and the Dean's Office at Fordham University for its generous financial support. The Q-Tof Ultima mass spectrometer (University of Illinois at Urbana-Champaign) was purchased in part with a grant from the NSF, Division of Biological Infrastructure (DBI0100085) and the Bruker MALDI-TOF/TOF UltrafleXtreme MS Spectrometer (New York University) was acquired through the support of the NSF (CHE-0958457).

\section{References}

1. Voit, B.; Komber, H.; Lederer, A. Hyperbranched Polymers: Synthesis and Characterization Aspects. In Synthesis of Polymers: New Structures and Methods; Schlueter, D. A.; Hawker, C. J.; Sakamoto, J., Eds.; Wiley-VCH Verlag GmbH \& Co: Weinheim, Germany, 2012; pp 701-740.

2. Irfan, M.; Seller, M. Ind. Eng. Chem. Res. 2010, 49, 1169-1196. doi:10.1021/ie900216r

3. Newkome, G. R.; Moorefield, C. N.; Vögtle, F. Dendrimers and Dendrons: Concepts, Synthesis, and Applications; Wiley-VCH Verlag GmbH: Weinheim, Germany, 2001. doi:10.1002/3527600612

4. Grayson, S. M.; Fréchet, J. M. J. Chem. Rev. 2001, 101, 3819-3867. doi:10.1021/cr990116h

5. Campagna, S.; Ceroni, P.; Puntoriero, F. Designing Dendrimers; Wiley-VCH Verlag GmbH: Weinheim, Germany, 2012.

6. Franc, G.; Kakkar, A. K. Chem. Soc. Rev. 2010, 39, 1536-1544. doi:10.1039/b913281n 
7. Walter, M. V.; Malkoch, M. Chem. Soc. Rev. 2012, 41, 4593-4609. doi:10.1039/c2cs35062a

8. Iha, R. K.; Wooley, K. L.; Nyström, A. M.; Burke, D. J.; Kade, M. J.; Hawker, C. J. Chem. Rev. 2009, 109, 5620-5686. doi:10.1021/cr900138t

9. Antoni, P.; Robb, M. J.; Campos, L.; Montanez, M.; Hult, A.; Malmström, E.; Malkoch, M.; Hawker, C. J. Macromolecules 2010, 43, 6625-6631. doi:10.1021/ma101242u

10. Montañez, M. I.; Campos, L. M.; Antoni, P.; Hed, Y.; Walter, M. V.; Krull, B. T.; Khan, A.; Hult, A.; Hawker, C. J.; Malkoch, M. Macromolecules 2010, 43, 6004-6013. doi:10.1021/ma1009935

11. Sridharan, V.; Muthusubramanian, S.; Sivasubramanian, S. Synth. Commun. 2004, 34, 1087-1096. doi:10.1081/SCC-120028641

12. Huang, Y.-M.; Ma, Q.-M. Liq. Cryst. 2010, 37, 1119-1126. doi:10.1080/02678292.2010.489156

13. Abdel-Magid, A. F.; Carson, K. G.; Harris, B. D.; Maryanoff, C. A.; Shah, R. D. J. Org. Chem. 1996, 61, 3849-3862. doi:10.1021/jo960057x

14. Dikusar, E. A.; Potkin, V. I.; Kozlov, N. G.; Yuvchenko, A. P. Russ. J. Gen. Chem. 2009, 79, 258-263. doi:10.1134/S1070363209020157

15. Li, C.-J.; Trost, B. M. Proc. Natl. Acad. Sci. U. S. A. 2008, 105, 13197-13202. doi:10.1073/pnas.0804348105

16. Hawker, C. J.; Fréchet, J. M. J. J. Chem. Soc., Perkin Trans. 11992 , 2459-2469. doi:10.1039/P19920002459

17. Pan, Y.; Ford, W. T. J. Org. Chem. 1999, 64, 8588-8593. doi:10.1021/jo991042c

18. Pan, Y.; Ford, W. T. J. Polym. Sci., Part A: Polym. Chem. 2000, 38, 1533-1543. doi:10.1002/(SICI)1099-0518(20000501)38:9<1533::AID-POLA17>3.0. CO;2-Q

19. Higuchi, M.; Shiki, S.; Yamamoto, K. Org. Lett. 2000, 2, 3079-3082. doi:10.1021/ol006241t

20. Takanashi, K.; Yamamoto, K. Org. Lett. 2007, 9, 5151-5154. doi:10.1021/ol701925a

21. See Supporting Information File 1 for the synthesis of benzaldehyde 7.

22. Spartan 14; Wavefunction, Inc.: Irvine, CA, USA, 2012.

23. Jansen, J. F. G. A.; de Brabander-van den Berg, E. M. M.; Meijer, E. W. Science 1994, 266, 1226-1229. doi:10.1126/science.266.5188.1226

24. Arkas, M.; Tsiourvas, D.; Paleos, C. M. Macromol. Mater. Eng. 2010, 295, 883-898. doi:10.1002/mame.201000219

25. Kannaiyan, D.; Imae, T. Langmuir 2009, 25, 5282-5285. doi:10.1021/la8039847

26. Lee, M. L.; Novotny, M. V.; Bartle, K. D. Analytical Chemistry of Polycyclic Aromatic Compounds; Academic Press: New York, 1981.

27. Polycyclic Aromatic Hydrocarbons: Chemistry and Carcinogenicity; Cambridge University Press: England, 1991. Harvey, R.G. Polycyclic Aromatic Hydrocarbons: Chemistry and Carcinogenicity. Cambridge University Press: England, 1991.

28. Arkas, M.; Allabashi, R.; Tsiourvas, D.; Mattausch, E.-M.; Perfler, R. Environ. Sci. Technol. 2006, 40, 2771-2777. doi:10.1021/es052290v

29. Arkas, M.; Tsiourvas, D.; Paleos, C. M. Chem. Mater. 2003, 15, 2844-2847. doi:10.1021/cm030214q

30. Arkas, M.; Eleades, L.; Paleos, C. M.; Tsiourvas, D. J. Appl. Polym. Sci. 2005, 97, 2299-2305. doi:10.1002/app.22026

31. See Supporting Information File 1 for details.

32. Arkas, M.; Tsiourvas, D. J. Hazard. Mater. 2009, 170, 35-42. doi:10.1016/j.jhazmat.2009.05.031
33. Ma, M.; Li, D. Chem. Mater. 1999, 11, 872-874. doi:10.1021/cm981090y

34. World Health Organization, Guidelines for Drinking-water Qualilty, 2nd ed. Addendum to Vol. 2. Health Criteria and Other and Other Supporting Information: Geneva 1998.

\section{License and Terms}

This is an Open Access article under the terms of the Creative Commons Attribution License

(http://creativecommons.org/licenses/by/2.0), which permits unrestricted use, distribution, and reproduction in any medium, provided the original work is properly cited.

The license is subject to the Beilstein Journal of Organic Chemistry terms and conditions:

(http://www.beilstein-journals.org/bjoc)

The definitive version of this article is the electronic one which can be found at: doi:10.3762/bjoc. 9.266 